\title{
New explanation for accelerated expansion and flat galactic rotation curves
}

\author{
Ahmad Sheykhi ${ }^{1,2, a}$ \\ ${ }^{1}$ Physics Department and Biruni Observatory, Shiraz University, Shiraz 71454, Iran \\ ${ }^{2}$ Institut für Physik, Universität Oldenburg, Postfach 2503, 26111 Oldenburg, Germany
}

Received: 27 November 2019 / Accepted: 26 December 2019 / Published online: 11 January 2020

(C) The Author(s) 2020

\begin{abstract}
Employing the non-additive Tsallis entropy, $S \sim$ $A^{\beta}$, for the large-scale gravitational systems, we disclose that in the cosmological scales both the Friedmann equation and the equation of motion for Newtonian cosmology get modified, respectively. We then derive the modified Newton's law of gravitation which is valid on large scales. We show that, in the relativistic regime, the modified Friedmann equation admits an accelerated expansion, for a universe filled with ordinary matter, without invoking any kind of dark energy, provided the non-extensive parameter is chosen $\beta<1 / 2$. In the non-relativistic regime, however, the modified Newton's law of gravitation can explain the flat galactic rotation curves without invoking particle dark matter provided $\beta \lesssim 1 / 2$. Our study may be regarded as an alternative explanation for the "dark side of the universe", through modification of the gravitational field equations.
\end{abstract}

\section{Introduction}

It is quite possible to speculate that the observed astrophysical and cosmological phenomena such as the late-time acceleration of the universe expansion, the flat rotation curves of spiral galaxies, the observed dynamics of the cluster of galaxies, the gravitational lensing, which cannot be understood through the standard Newton and Einstein theories of gravitation, are just weaknesses of the underlying theory of gravity. If that is true, one may expect that such phenomena are just geometrical effects arising due to the flaw of the theory. Therefore, one should be capable to explain the observed cosmological phenomena through modifying the underlying theory of gravity. Many attempts have been done to find possible solutions for the puzzles of accelerated expansion and flat galactic rotation curves from geometrical perspective. In particular, over the past years, modified theories of grav-

a e-mail: asheykhi@shirazu.ac.ir ity have gained considerable attention. Among them, perhaps the one best known, is $f(R)$ gravity, which tries to explain early inflation, late-time acceleration and even the flat galactic rotation curves through modification of the EinsteinHilbert action (see [1-16] and the references therein). For a recent comprehensive review of different modified gravity techniques which contains all the necessary information in the context of cosmology, emphasizing inflation, bouncing cosmology and late-time acceleration, we refer to [17].

Another attempt for explanation of the dark matter puzzle, through a non-relativistic model, is modified Newtonian dynamics (MOND) $[18,19]$, which proposed to address the flat rotation curves of spiral galaxies through modifying Newton's law of gravitation. Although MOND theory can explain the flat galactic rotation curves, it suffers from several problems. First of all, it is problematic to embed MOND theory within a more comprehensive relativistic theory of gravity and, hence, its theoretical origin remains unclear. Second, it predicts that the individual halo associated with a galaxy is infinite in extent, while recent galaxy-galaxy lensing results suggest that galaxy halos may have a maximum extent of about $0.5 \mathrm{Mpc}$ [20]. Some authors have also tried to explain the flat rotation curves through modification of Einstein gravity [21-26].

In this paper, we would like to propose an alternative perspective for tackling the problem of accelerated expansion as well as the flat galactic rotation curves through modifying the Friedmann equation and Newton's law of gravitation, using a thermodynamic argument. In 1902 Gibbs pointed out that, in systems where the partition function diverges, the standard Boltzmann-Gibbs theory is not applicable, and largescale gravitational systems are known to fall within this class. Hence, the usual Boltzmann-Gibbs additive entropy must be generalized to the non-additive (non-extensive) entropy (the entropy of the whole system is not necessarily the sum of the entropies of its sub-systems) [27-29]. In 1988 Tsallis generalized standard thermodynamics to non-extensive thermody- 
namics, which can be applied in all cases, and still possessing standard Boltzmann-Gibbs theory as a limit [27]. Based on this, and using statistical arguments, Tsallis and Cirto argued that the entropy of a black hole does not obey the area law and can be modified as [30]

$S=\gamma A^{\beta}$,

where $A$ is the horizon area, $\gamma$ is an unknown constant, and $\beta$ is known as the non-extensive parameter.

On the other hand, a thermodynamical interpretation of the gravitational field equations, based on the profound connection between the first law of thermodynamics on the boundary, and the gravitational field equations in the bulk, is now an established fact $[31,32]$. It was argued that, given an entropy expression at hand, in any gravity theory, one can rewrite the Friedmann equations of the Friedmann-Robertson-Walker (FRW) universe in the form of the first law of thermodynamics on the apparent horizon and vice versa [33-50]. Recently, for the case the entropy of the gravitational system is in the form of the non-extensive Tsallis entropy, the thermodynamical interpretation of the gravitational field equations and the effects of the non-extensive parameter in the context of cosmology have aroused a lot of interests. It was shown that the non-extensive parameter changes the strength of the gravitational constant and consequently the energy density of the dark components of the universe, requiring more (less) dark energy to provide the observed late-time universe acceleration [51,52]. In the context of the non-extensive Kaniadakis statistics $[53,54]$, the Jeans length was investigated and the results were compared with the Jeans length obtained in the non-extensive Tsallis statistics [55]. The cosmological scenarios based on the non-extensive Tsallis entropy have been explored in [56]. It was shown that the universe exhibits the usual thermal history, with the sequence of matter and dark-energy eras, and depending on the value of nonextensive parameter, this scenario may entail a variety of dark-energy models [56]. Taking the entropy associated with the apparent horizon of the FRW universe in the form of Tsallis entropy, and assuming the first law of thermodynamics, $\mathrm{d} E=T \mathrm{~d} S+W \mathrm{~d} V$, to hold on the apparent horizon, the modified Friedmann equations describing the dynamics of the universe with any spatial curvature were extracted [57]. It was argued that with an appropriate choice for the nonextensive parameter, this model is capable to reproduce the late-time cosmic acceleration, as well as the early deceleration, in the absence of dark energy [57]. The studies on the Tsallis cosmology were also generalized to the case with variable non-extensive parameter [58]. It was shown that the extra terms, arising from the non-extensive entropy, can play the role of an effective dark energy describing the evolution of the universe from the early epoch to late-time acceleration [58]. More recently, it was shown that it is quite possible to establish a correspondence between the non-extensive
Tsallis cosmology and cosmology with a fluid with redefined equation of state for both constant and variable nonextensive parameter [59]. In this viewpoint, the effective fluid can derive successfully not only the present acceleration but also the early inflation without spoiling the correct late-time acceleration [59].

In this paper, we consider the Tsallis entropy given in (1) as the entropy expression of the gravitational systems, and disclose that, in the relativistic regime, it could modify the Friedmann equation and the resulting equation is capable to provide, naturally, the late-time accelerated expansion without invoking any kind of dark energy. On the non-relativistic regime, however, the modification to Newton's law of gravitation leads to an explanation of the flat rotation curves of galaxies without need of particle dark matter. Let us stress the similarity and difference of the present work with alternative theories of gravity, in particular $f(R)$ gravity. First of all, similar to our work, $f(R)$-gravity theories can also explain the dark side of the universe through modification of the geometrical part of the Einstein gravity. For a comprehensive and excellent review on $f(R)$-gravity we refer to [10], where it was shown that $f(R)$ theory can be considered as a unified description for the history of the universe from the early-time inflation to the late-time acceleration. However, in $f(R)$-gravity, in order to establish a correspondence between the field equations and the first law of thermodynamics, a treatment with non-equilibrium thermodynamics is required $[32,43]$. In this case the first law of thermodynamics acquires an additional entropy production term generated internally due to the non-equilibrium treatment of the system. This is in contrast to the Einstein gravity, and also its modification when the entropy of the system is modified as a non-extensive Tsallis entropy. It was shown that, in the presence of non-extensive entropy, the Friedmann equation of FRW cosmology in a nonflat $[56,57]$ and flat $[58,59]$ universe can be deduced from the first law of thermodynamics, on the apparent/Hubble horizon, in a complete equilibrium situation. This is one of the main differences between our work and $f(R)$ gravity. Besides, for an explanation of the flat galactic rotation curves, we apply the non-relativistic modified Newton's law of gravity based on Tsallis entropy, while in $f(R)$-gravity, the problem is addressed through modifying General Relativity $[6,9,13]$.

This paper is outlined as follows. In the next section, we show how the modified Friedmann equation in Tsallis cosmology leads to the late-time accelerated expansion in the universe filled with ordinary baryonic matter. In Sect. 3, we derive the modified Newton's law of gravitation based on the Tsallis entropy. In Sect. 4, we show that the modified Newton's law of gravitation can also be extracted from an entropic force scenario. In Sect. 5, we employ the modified Newton's law of gravity and disclose that it can explain the flat rotation curves of spiral galaxies. In this viewpoint, dark matter has 
only a geometrical effect that originates with a modification of gravity. The last section is devoted to our conclusions.

\section{Accelerated universe in Tsallis cosmology}

Let us start by deriving the modified Friedmann equation based on the non-extensive Tsallis entropy from the first law of thermodynamics. This problem was already studied in [56-59], but since it provides a basis for the next sections, for completeness, we briefly review the derivation here. Following [57], we assume that the non-extensive Tsallis entropy affects the geometry part of the Friedmann equations and hence we keep the energy content of the universe in the form of a standard perfect fluid. It is important to note that a different viewpoint was recently adopted in [59] by assuming that the non-extensive entropy modifies the energy density and hence the pressure of the universe. As a result, one should redefine the equation of state of the perfect fluid [59].

Suppose the background of spacetime is given by the FRW geometry,

$\mathrm{d} s^{2}=h_{\mu \nu} \mathrm{d} x^{\mu} \mathrm{d} x^{\nu}+\tilde{r}^{2}\left(\mathrm{~d} \theta^{2}+\sin ^{2} \theta \mathrm{d} \phi^{2}\right)$.

In the above line element $\tilde{r}=a(t) r, x^{0}=t, x^{1}=r$, and $h_{\mu \nu}=\operatorname{diag}\left(-1, a^{2} /\left(1-k r^{2}\right)\right)$ stands for the metric of a two dimensional subspace. We also assume our universe is bounded by an apparent horizon with radius $\tilde{r}_{A}=1 / \sqrt{H^{2}+k / a^{2}}$. Using the definition of the surface gravity $\kappa$ for the apparent horizon, it is easy to show that the temperature on the apparent horizon can be given by the relation $[40]$

$T=\frac{\kappa}{2 \pi}=-\frac{1}{2 \pi \tilde{r}_{A}}\left(1-\frac{\dot{\tilde{r}}_{A}}{2 H \tilde{r}_{A}}\right)$.

Suppose the energy-momentum tensor of the universe is $T_{\mu \nu}=(\rho+p) u_{\mu} u_{\nu}+p g_{\mu \nu}$, the conservation equation, $\nabla_{\mu} T^{\mu \nu}=0$, for the FRW geometry implies the continuity equation as $\dot{\rho}+3 H(\rho+p)=0$. Because our universe is expanding, as a thermodynamical system, work should be done due to the volume change of the system. The density of this work, on the FRW background, is given by $[60,61]$

$W=\frac{1}{2}(\rho-p)$.

Finally, we propose the first law of thermodynamics holds on the apparent horizon,

$\mathrm{d} E=T \mathrm{~d} S+W \mathrm{~d} V$.

Note that, for a pure de Sitter space, $\rho=-p$, the first law reduces to $\mathrm{d} E=T \mathrm{~d} S-p \mathrm{~d} V$. If we denote the total energy of the universe $E=\rho V$ where $V=\frac{4 \pi}{3} \tilde{r}_{A}^{3}$, after differentiating, we arrive at $\mathrm{d} E=4 \pi \tilde{r}_{A}^{2} \rho \mathrm{d} \tilde{r}_{A}+\frac{4 \pi}{3} \tilde{r}_{A}^{3} \dot{\rho} \mathrm{d} t$

Substituting $\dot{\rho}$ from the continuity equation yields

$\mathrm{d} E=4 \pi \tilde{r}_{A}^{2} \rho \mathrm{d} \tilde{r}_{A}-4 \pi H \tilde{r}_{A}^{3}(\rho+p) \mathrm{d} t$.

Then we should consider the evolution of the Tsallis entropy which we assume has the form (1). Taking the differential of the Tsallis entropy, we find

$\mathrm{d} S=8 \pi \gamma \beta\left(4 \pi r_{A}^{2}\right)^{\beta-1} \tilde{r}_{A} \mathrm{~d} \tilde{r}_{A}$.

Inserting Eqs. (3), (4), (7) and (8) in the first law (5), we obtain

$\frac{\gamma \beta}{\pi \tilde{r}_{A}^{3}}\left(4 \pi \tilde{r}_{A}^{2}\right)^{\beta-1} d \tilde{r}_{A}=H(\rho+p) \mathrm{d} t$.

Using the continuity equation, we get

$-\frac{2}{\tilde{r}_{A}^{3}}\left(4 \pi \tilde{r}_{A}^{2}\right)^{\beta-1} \mathrm{~d} \tilde{r}_{A}=\frac{2 \pi}{3 \gamma \beta} \mathrm{d} \rho$.

Integrating yields

$\frac{1}{\tilde{r}_{A}^{4-2 \beta}}=\frac{2 \pi(2-\beta)}{3 \gamma \beta}(4 \pi)^{1-\beta} \rho$,

where the constant of integration is set equal to zero. Finally, we define the constant $\gamma$ by

$\gamma \equiv \frac{2-\beta}{4 \beta L_{p}^{2}}(4 \pi)^{1-\beta}$

where $L_{p}=\sqrt{G \hbar / c^{3}}$ is the Planck length. Since the entropy is positive definite $(\gamma>0)$, the above definition also implies $\beta<2$. After using the definition of $\tilde{r}_{A}$, Eq. (11) immediately transforms to

$$
\left(H^{2}+\frac{k}{a^{2}}\right)^{2-\beta}=\frac{8 \pi L_{p}^{2}}{3} \rho .
$$

In this way we derive the modified Friedmann equation which describes the evolution of the universe in Tsallis cosmology based on the non-extensive Tsallis entropy. When $\beta=1$, the standard Friedmann equation is recovered. The second Friedmann equation can be easily derived by combining the continuity equation with Eq. (13). Now we want to study the cosmological consequences of the modified Friedmann equation obtained. It is a matter of calculation to show that the second derivative of the scale factor satisfies the following equation:

$\frac{\ddot{a}}{a}\left(H^{2}+\frac{k}{a^{2}}\right)^{1-\beta}=-\frac{4 \pi L_{p}^{2}}{3(2-\beta)}[(2 \beta-1) \rho+3 p]$.

Therefore, the accelerated expansion $(\ddot{a}>0)$ can be achieved provided

$(2 \beta-1) \rho+3 p<0, \Rightarrow \omega<\frac{1-2 \beta}{3}$, 
where $\omega=p / \rho$ denotes the equation of state parameter. Condition (15) has interesting consequences. Let us consider it carefully in two cases. In the first case, where $\beta \geq 1 / 2$, we always have $\omega<0$ as a condition for an accelerated universe. In the second case, where $\beta<1 / 2$, it is quite possible to have $\omega \geq 0$, while our universe is still accelerating $(\ddot{a}>0)$. This is a very interesting result which confirms that, in the framework of Tsallis cosmology, the current acceleration of the universe expansion can be understood, in the presence of ordinary matter with $w \geq 0$. Precisely speaking, we can consider a universe filled with pressureless baryonic matter, and it still enjoys an accelerated expansion, without invoking any dark companion for its matter/energy content.

The above discussion can also be confirmed by looking explicitly at the scale factor. Assuming a flat FRW universe filled with pressureless matter $(p=0)$, the Friedmann equation (13) admits the solution $a(t) \sim t^{(4-2 \beta) / 3}$. This implies that $\ddot{a}(t) \propto(2-\beta)(1-2 \beta) t^{-(2+2 \beta) / 3}$, where the constant of proportionality is also a positive definite [57]. Thus, for $\beta<1 / 2$ we have an accelerated universe ( $\ddot{a}>0$ ), in accordance with condition (15). It was also argued that not only the accelerated expansion but also the early deceleration as well as the age problem of the universe can be circumvented automatically in the context of Tsallis cosmology without invoking an additional dark component of the energy [57].

We emphasize here that the authors of [58,59] argued that the modified Friedmann equations, derived from Tsallis entropy, can reproduce the late-time acceleration provided one takes an effective dark energy [58] or a redefined fluid with generalized equation of state [59]. They also established a correspondence between the modified cosmology through non-extensive thermodynamics and the holographic dark-energy model as well as $f(R)$ gravity [58]. It is important to note that, for a derivation of the Friedmann equations, the authors of $[58,59]$ assumed the first law of thermodynamics on the Hubble horizon as $\mathrm{d} Q=T \mathrm{~d} S$, where $\mathrm{d} Q$ is the heat flux crossing the horizon, and the spacetime is spatially flat. Meanwhile, here we derived the modified Friedmann equation for any spatial curvature by assuming that $\mathrm{d} E=T \mathrm{~d} S+W \mathrm{~d} V$ holds on the apparent horizon. Another difference between our work and $[58,59]$ is that we could reproduce the late-time acceleration in the presence of ordinary matter, without needing to redefine the fluids or taking into account effective dark energy.

Finally, it is worthy to note that the correspondence between Tsallis cosmology and the standard Firedmann equation with the fluids of the redefined equation of state established in [59] comes from the fact that the field equations of General Relativity, and hence the Friedmann equations, relate the geometry of spacetime to its energy content. Thus, any modification of the geometry can be translated to a modification of the energy content and vice versa. In Tsallis cosmology, based on non-extensive entropy, it is quite pos- sible to consider the modification in the geometry part of the gravitational field equation, and keep the energy content as standard perfect fluid [57]. This is reasonable, because the definition of the entropy is based on the area (geometry) of the system, and thus any modification in entropy should affect the geometry part of the field equations and vice versa $[32,44,45]$.

\section{Modified Newton's law of gravity}

In this section we first derive the equation of motion describing the evolution of the universe in Newtonian cosmology. Using this equation, we then derive the modified Newton's law of gravitation which is based on the non-extensive Tsallis entropy. We start from the Friedmann equation (13) by taking the time derivative of it. We arrive at

$$
(2-\beta) \frac{\ddot{a}}{a}\left(\dot{a}^{2}+k\right)^{1-\beta}=-\frac{4 \pi L_{p}^{2}}{3}[(2 \beta-1) \rho+3 p] a^{2-2 \beta} .
$$

When $\beta=1$, it reduces to

$$
\frac{\ddot{a}}{a}=-\frac{4 \pi L_{p}^{2}}{3}(\rho+3 p) \text {, }
$$

which is the evolutionary equation for the scale factor in standard cosmology. We also assume in the Newtonian cosmology the spacetime is Minkowskian with $k=0$, and work in the unit $\hbar=c=1$, and so $L_{p}^{2}=G$. Therefore, Eq. (16) reduces to

$$
(2-\beta) \frac{\ddot{a}}{a}=-\frac{4 \pi G}{3}[(2 \beta-1) \rho+3 p]\left(\frac{a}{\dot{a}}\right)^{2-2 \beta} .
$$

We consider a compact spatial region $V$ with a compact boundary $\mathcal{S}$, which is a sphere with physical radius $R=$ $a(t) r=H^{-1}$, where $r$ is a dimensionless co-moving coordinate which remains constant for any cosmological object partaking in free cosmic expansion. The active gravitational mass in General Relativity, inside the volume $V$, is defined as [42]

$\mathcal{M}=2 \int_{V} \mathrm{~d} V\left(T_{\mu \nu}-\frac{1}{2} T g_{\mu \nu}\right) u^{\mu} u^{\nu}$.

A simple calculation gives

$\mathcal{M}=(\rho+3 p) \frac{4 \pi}{3} R^{3}$.

In order to transform from General Relativity to the Newtonian gravity, we also replace the active gravitational mass $\mathcal{M}$ with the total mass $M=\rho V=4 \pi \rho R^{3} / 3$. This is equal to transforming $\rho+3 p \rightarrow \rho$ in Eq. (18),

$(2-\beta) \frac{\ddot{a}}{a}=-\frac{4 \pi G}{3} \rho(2 \beta-1)\left(\frac{a}{\dot{a}}\right)^{2-2 \beta}$. 
This is nothing but the modified dynamical equation describing the evolution of the universe in Newtonian cosmology. In the limiting case where $\beta=1$, we find

$\frac{\ddot{a}}{a}=-\frac{4 \pi G}{3} \rho$,

which is the standard equation of motion in Newtonian cosmology. On the other hand, the acceleration of a test particle $m$ near the surface $\mathcal{S}$ can be written

$\ddot{R}=\ddot{a} r=F / m$,

where $F$ is the gravitational force between $m$ from $M$. Equating $\ddot{a}$ in Eqs. (21) and (23), we find

$F=-\left(\frac{2 \beta-1}{2-\beta}\right) \frac{4 \pi G}{3} \rho m R\left(\frac{a}{\dot{a}}\right)^{2-2 \beta}$.

Using the fact that $R=1 / H=a / \dot{a}$ and $\rho=M / V$, the above equation can be rewritten as

$F=-\left(\frac{2 \beta-1}{2-\beta}\right) \frac{G M m}{R^{2 \beta}}$.

In this way we derive the modified Newton's law of gravity based on the non-extensive Tsallis entropy. When $\beta=1$, one recovers the well-known Newton law of gravitation.

\section{Newton's law from entropic force}

Now we want to employ the idea of entropic gravity proposed by Verlinde [62] and show that Newton's law of gravity gets modified when the entropy of the system is in the form of Tsallis entropy. According to Verlinde's hypothesis, gravity can be regarded as an entropic force caused by changes in the information associated with the positions of material bodies. Using first principles, namely the equipartition law of energy in statistical mechanics together with the holographic principle, he derived Newton's law of gravitation, the Poisson equation and in the relativistic regime the Einstein field equations of General Relativity [62]. Although the idea was already addressed by Padmanabhan [63] that gravity has a statistical origin, and in particular, one can use the equipartition law of energy to provide a thermodynamic interpretation of gravity, the notion that gravity is not a fundamental force and can be identified as an entropic force was first pointed out by Verlinde [62].

According to Verlinde, when a test particle moves away from the holographic screen, it will experience an effective force equal to

$F \triangle x=T \triangle S$,

where $T$ and $\triangle S$ are, respectively, the temperature and the entropy of the surface and $\Delta x$ is the displacement of the test particle from the holographic screen. Thus, in order to have a non-vanishing entropic force, we need to have a non-zero temperature.

Suppose that a holographic screen, which by assumption is a spherically symmetric surface $\mathcal{S}$ with area $A=4 \pi R^{2}$, is a storage device for information and the holographic principle holds. It is natural to assume that the total number of bits, $N$, is proportional to the area/entropy, $N \sim A \sim S$. The total energy $E$ of the system inside the holographic screen is distributed on these bits and thus the temperature on the surface is given by the equipartition law of energy

$E=\frac{1}{2} N k_{B} T \Rightarrow T=\frac{2 E}{N k_{B}}$.

We also assume that the total energy of the system can be written as $E=M c^{2}$ where $M$ is the total mass distribution inside the holographic spherically symmetric screen which is uniformly distributed [62]. The surface $\mathcal{S}$ is located between the test mass $m$ and the mass distribution $M$, and the test mass is assumed to be very close to the surface compared to its reduced Compton wavelength $\lambda_{m}=\hbar /(m c)$. Finally, we write the number of bits on the holographic surface as

$N=4 S=\frac{2-\beta}{\beta G}(4 \pi)^{1-\beta} A^{\beta}$,

where we have assumed $S=\gamma A^{\beta}$ and we used the definition (12). Following [62], we postulate a change of the entropy associated with the information on the holographic screen equaling

$\triangle S=2 \pi k_{B}$ when $|\Delta x|=\eta \lambda_{m}$,

where $\eta$ is a constant which should be defined later. We also assume the entropy gradient points radially from the outside of the surface to inside. Combining Eqs. (27), (28) and (29) with (26) and working in units $\hbar=c=1$, we arrive at

$F=-\frac{\beta}{\eta(2-\beta)} \frac{G M m}{R^{2 \beta}}$.

Finally, we redefine $\eta=\beta /(2 \beta-1)$ and rewrite the above equation in the form

$F=-\frac{(2 \beta-1)}{(2-\beta)} \frac{G M m}{R^{2 \beta}}$.

This is the modified Newton law of gravitation derived from the viewpoint that gravity is an entropic force, by assuming that the entropy of a gravitational system is in the form of the non-additive Tsallis entropy. It is clear that our result from the entropic force approach coincides with the result obtained in the previous section. Our investigation shows that with the correction to the area law, Newton's law of gravitation gets modified, accordingly.

It is important to note that in order to arrive at the standard Newton law of gravitation, one should take the limit $\beta \rightarrow 1$, which corresponds to the area law for the entropy. This implies that the modified Newton's law derived from the 
Tsallis entropy holds on the large-scale gravitational system, namely at outside the galaxies. This is consistent with the argument which states that in systems with diverging partition function, such as large-scale gravitational systems, the standard Boltzmann-Gibbs theory cannot be applied and one needs to use non-extensive Tsallis thermodynamics, which still possesses standard Boltzmann-Gibbs theory as a limit [27-29]. Clearly, $\beta \rightarrow 1$ is the limiting case of our theory. Note that inside the galaxy, the standard Newton's law can correctly predicate the orbital speed of star and the problem of flat rotation curves (the difference between observation and theory) appears only outside the galaxies. On cosmological scales, including outside the galaxies, the entropy still obeys the non-extensive one, thus that Tsallis cosmology holds in the theory is realistic.

In the next section, we shall use this modified Newton's law of gravity and show that it is indeed capable of explaining the flat rotation curves of spiral galaxies.

\section{Explanation of the galaxies rotation curves}

There are a lot of observational data which confirm that the rotational velocity curves of all spiral galaxies are proportional to the distance from the center, $v \propto r$, inside the galaxy, and rotation curves usually remain almost flat far from galactic centers, typically beyond $30-40 \mathrm{kpc}$. Inside the galaxy, however, these observations can be completely understood via Newtonian gravity. Unfortunately, outside a spiral galaxy, Newton's law of gravitation is not capable of explaining the rotational curves, and there is indeed a contradiction between observation and the prediction of the theory, since Newton's law of gravitation predicts that objects that are far from the galaxy center have lower velocities $v \propto r^{-1 / 2}$, while observations imply that the velocity curves flattened out to $v \simeq$ constant. It is well established that the baryonic matter of galaxies does not provide sufficient gravitation to explain the observed dynamics of the systems. The most widely adopted way to resolve these difficulties is the dark matter hypothesis, which suggests that the mass of galaxies continues to grow even when there is no luminous component to account for this increase. According to this hypothesis, all visible galaxies are surrounded by massive nonluminous matter. Besides the dark matter proposal, alternative theories of gravitation have also been speculated and debated on for justification of the flat rotation curves. As we mentioned in the introduction, Milgrom $[18,19]$ tried to explain the flat rotation curves of galaxies, through modifying Newton's law of gravity (MOND), and he represents it as a geometrical effect. However, MOND theory suffers from the flaws as regards the theoretical origin.
Here we tackle this problem by modifying Newton's law of gravity based on the Tsallis entropy. Interestingly enough, we will show that the modified Newton's law at large scales, given in Eq. (31), is capable to naturally explain the flat rotation curves of spiral galaxies. We postulate that $\beta=1$ inside the galaxies where the usual Newton's law holds, and $\beta<1 / 2$ at far distances, at the galaxy's outer skirts. This implies that inside a galaxy, and at distance $r$ from its center, we must have

$a=\frac{v^{2}}{r}=\frac{G M(r)}{r^{2}} \rightarrow v \propto r$,

where we have used the fact that inside the galaxy the total mass which contributes to the velocity is $M(r)=4 \pi r^{3} \rho / 3$. However, at distances $r$ large enough for there to be no luminous galactic component there are indications that we have $M(r)=M \simeq$ constant, and thus from Eq. (31) we have

$$
\begin{aligned}
& \frac{v^{2}}{r}=\left(\frac{1-2 \beta}{2-\beta}\right) \frac{G M}{r^{2 \beta}} \\
& \Rightarrow v(r)=\sqrt{\left(\frac{1-2 \beta}{2-\beta}\right) G M r^{1-2 \beta}} .
\end{aligned}
$$

Clearly, $\beta \neq 1 / 2$, and in particular we should have $\beta<1 / 2$, but for later convenience, we assume the values of $\beta$ to be close to $1 / 2$. In order to have a better understanding of the behavior of $v$ in terms of distance, let apply the above formula for a typical spiral galaxy. For this purpose, we assume $M=M_{i}$ is the total mass of the galaxy. We also set the Newtonian gravitational constant $G \simeq 6.674 \times 10^{-11} \mathrm{~m}^{3} \mathrm{~kg}^{-1} \mathrm{~s}^{-2}$ and take the mass of the Sun $M_{\odot} \simeq 10^{30} \mathrm{~kg}$. Since the mass of a typical galaxy is of order $\sim 10^{9} M_{\odot}$, in these figures we assume the mass of a typical galaxy ranges in $8 \times 10^{9} M_{\odot}<M_{i}<107 \times 10^{9} M_{\odot}$, which are reasonable values, at least for small spiral galaxies. We have plotted Figs. 1, 2 and 3 for different values of the mass $M$ and the non-extensive parameter $\beta$. In all figures, we observe that the speed of a test particle increases at small distance (inside galaxy) and tends to an almost constant value at far distance, at the galaxy's outer skirts. From Figs. 1 and 2 we see that, for a fixed value of $\beta<1 / 2$, but close to it, at any distance, the orbital speed increases with increasing the mass of the galaxy. Also, from Fig. 3 one can see that, for a fixed value of $M$, at any distance, the orbital speed increases with increasing non-extensive parameter $\beta$. These figures are compatible with astrophysical data [64-66]. Note that here we have presented the ideas, and have shown how the modified Newton's law of gravity given in (33), at large distance, can explain the flat galactic rotation curves. We leave the details of data fitting of this model with observations for future studies. 


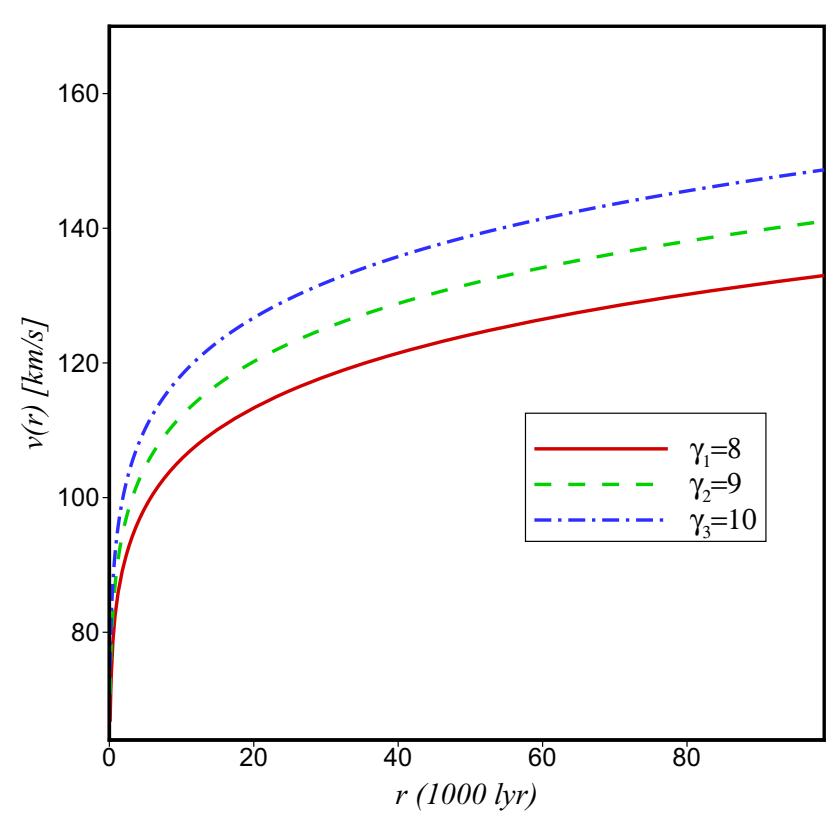

Fig. 1 The speed of a test particle around a spiral galaxy in terms of the distance for $\beta=0.40$ and different values of galaxy mass $M_{i}=$ $\gamma_{i} \times 10^{9} M_{\odot}$

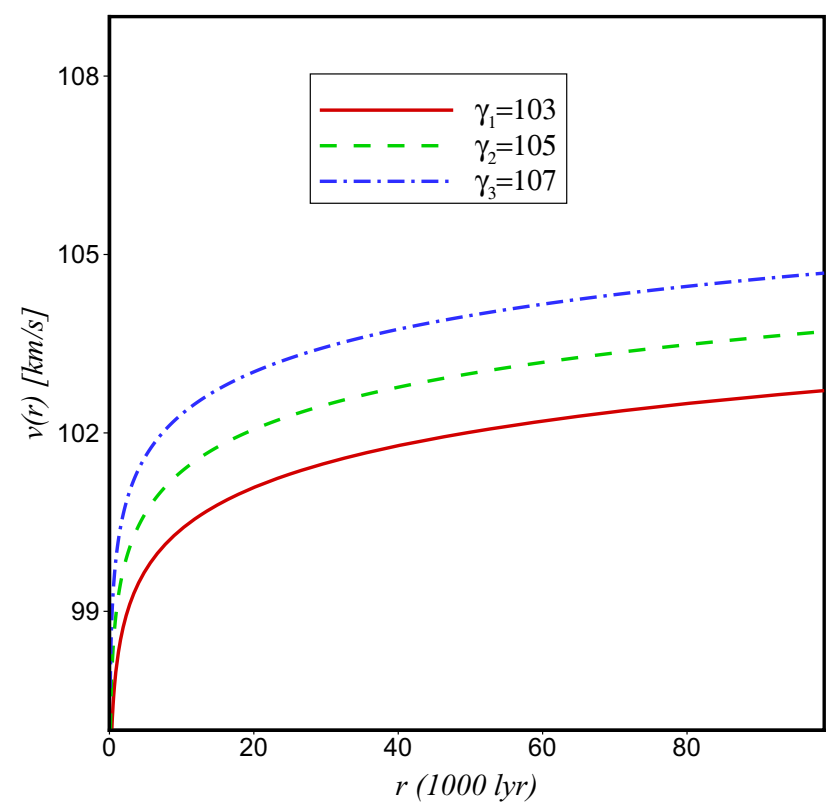

Fig. 2 The speed of a test particle around a spiral galaxy in terms of distance for $\beta=0.49$ and different values of galaxy mass $M_{i}=$ $\gamma_{i} \times 10^{9} M_{\odot}$

\section{Conclusions}

Using the non-extensive Tsallis entropy for large-scale gravitational systems, we disclosed that on the relativistic cosmological background the Friedmann equations describing the evolution of the FRW universe get modified, accordingly. Starting from the first law of thermodynamics on the appar-

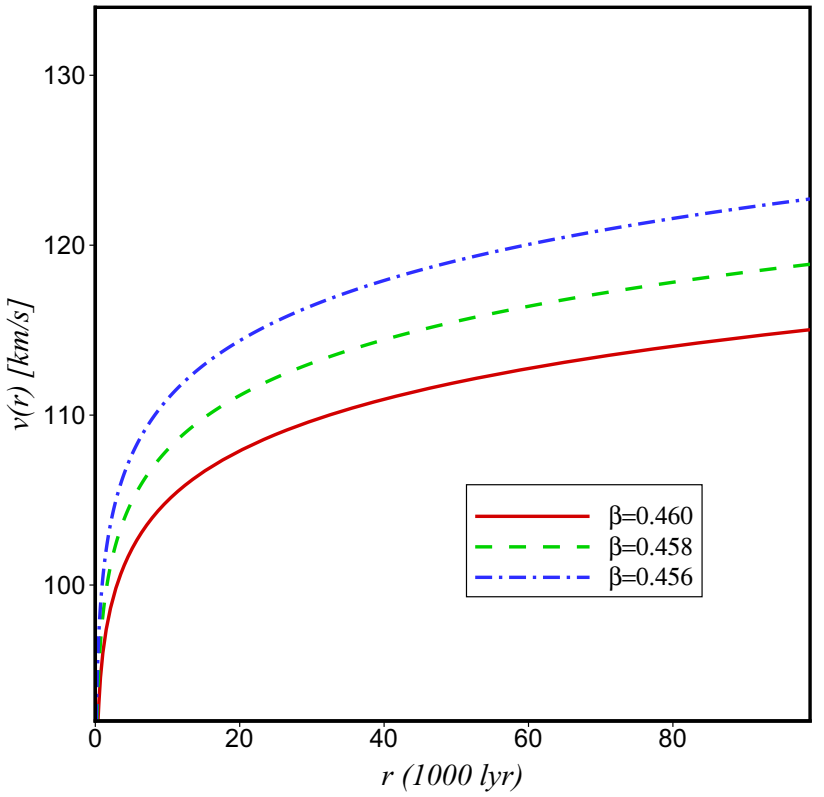

Fig. 3 The speed of a test particle around a spiral galaxy for a typical galaxy with mass $M=25 \times 10^{9} M_{\odot}$

ent horizon, we derived the modified Friedmann equation. We observed that when the non-extensive parameter satisfies $\beta<1 / 2$, the late-time acceleration of the cosmic expansion can be achieved in the presence of ordinary matter. This implies that one may consider a universe filled with baryonic matter, and that still enjoys an accelerated expansion without invoking any dark companion for its matter/energy content. On the other hand, in the regime of non-relativistic gravity, one is able to reproduce the equation of motion describing the evolution of the universe in Newtonian cosmology. We then derived the modified Newton's law of gravitation based on the Tsallis entropy from two approaches. The first one is an inverse approach by starting from Newtonian cosmology, and the second one is extracted through an entropic force scenario [62]. We showed that the two approaches lead to the same results. Interestingly enough, we observed that flat galactic rotation curves can be explained, through a modified Newton's law of gravitation, provided $\beta \lesssim 1 / 2$, without need of particle dark matter.

Finally, we would like to stress that in this work, in contrast to $f(R)$ gravity theories which try to explain the flat galactic rotation curves through modifying Einstein gravity, we implemented the problem in the context of non-relativistic modified Newton's law of gravity. In a sense our work can be located in the MOND theories category, however, the advantage of our work is that its theoretical origin is well established.

Acknowledgements I am grateful to anonymous referee for valuable comments which helped me improve the paper significantly. I thank Shiraz University Research Council. My special thanks go to Jutta Kunz for helpful discussions and the University of Oldenburg, for hospitality. 
Data Availability Statement This manuscript has no associated data or the data will not be deposited. [Author's comment: Most of the calculations have been done by the hand, so there is no data files available for this research.]

Open Access This article is licensed under a Creative Commons Attribution 4.0 International License, which permits use, sharing, adaptation, distribution and reproduction in any medium or format, as long as you give appropriate credit to the original author(s) and the source, provide a link to the Creative Commons licence, and indicate if changes were made. The images or other third party material in this article are included in the article's Creative Commons licence, unless indicated otherwise in a credit line to the material. If material is not included in the article's Creative Commons licence and your intended use is not permitted by statutory regulation or exceeds the permitted use, you will need to obtain permission directly from the copyright holder. To view a copy of this licence, visit http://creativecomm ons.org/licenses/by/4.0/.

Funded by $\mathrm{SCOAP}^{3}$.

\section{References}

1. S. Capozziello, Int. J. Mod. Phys. D 11, 483 (2002)

2. S. Nojiri, S.D. Odintsov, Phys. Rev. D 68, 123512 (2003). arXiv:hep-th/0307288

3. S. Nojiri, S.D. Odintsov, Phys. Lett. B 631, 1 (2005). arXiv:hep-th/0508049

4. S. Nojiri, S.D. Odintsov, Phys. Rev. D 74, 086005 (2006). arXiv:hep-th/0608008

5. R. Zaregonbadi, M. Farhoudi, N. Riazi, Phys. Rev. D 94, 084052 (2016). arXiv:1608.00469

6. Y. Sobouti, Astron. Astrophys. 464, 921 (2007)

7. G. Cognola, E. Elizalde, S. Nojiri, S.D. Odintsov, L. Sebastiani, S. Zerbini, Phys. Rev. D 77, 046009 (2008). arXiv:0712.4017

8. T.P. Sotiriou, V. Faraoni, Rev. Mod. Phys. 82, 451 (2010). arXiv:0805.1726

9. C.G. Boehmer, T. Harko, F.S.N. Lobo, Astropart. Phys. 29, 386 (2008). arXiv:0709.0046

10. S. Nojiri, S.D. Odintsov, Phys. Rep. 505, 59 (2011). arXiv:1011.0544

11. S. Capozziello, M. De Laurentis, Phys. Rep. 509, 167 (2011). arXiv: 1108.6266

12. S. Nojiri, S.D. Odintsov, Mod. Phys. Lett. A 29(40), 1450211 (2014). arXiv: 1408.3561

13. F. Shojai, A. Shojai, Gen. Relat. Gravit. 46(4), 1704 (2014). arXiv: 1404.0299

14. J.-H. He, A.J. Hawken, B. Li, L. Guzzo, Phys. Rev. Lett. 115, 071306 (2015). arXiv: 1501.00846

15. S.D. Odintsov, V.K. Oikonomou, Phys. Rev. D 99, 104070 (2019). arXiv: 1905.03496

16. S.D. Odintsov, V.K. Oikonomou, Phys. Rev. D 99, 064049 (2019). arXiv: 1901.05363

17. S. Nojiri, S.D. Odintsov, V.K. Oikonomou, Phys. Rep. 692, 1 (2017). arXiv: 1705.11098

18. M. Milgrom, Astrophys. J 270, 365 (1983)

19. M. Milgrom, Astrophys. J. 270, 371 (1983)

20. H. Hoekstra, H.K.C. Yee, M.D. Gladders, arXiv:astro-ph/0109514

21. Y. Sobouti, arXiv:0810.2198

22. Y. Sobouti, Dark Matter in Astrophysics and Particle Physics, vol. 356 (2009)
23. A.H. Chamseddine, V. Mukhanov, Mimetic Dark Matter. JHEP 1311, 135 (2013)

24. R. Myrzakulov et al., Class. Quantum Gravity 33, 125005 (2016). arXiv: 1510.02284

25. S. Vagnozzi, Class. Quantum Gravity 34, 185006 (2017). arXiv: 1708.00603

26. A. Sheykhi, S. Grunau, arXiv:1911.13072

27. C. Tsallis, J. Stat. Phys. 52, 479 (1988)

28. M.L. Lyra, C. Tsallis, Phys. Rev. Lett. 80, 53 (1998)

29. G. Wilk, Z. Wlodarczyk, Phys. Rev. Lett. 84, 2770 (2000). arXiv:hep-ph/9908459

30. C. Tsallis, L.J.L. Cirto, Eur. Phys. J. C 73, 2487 (2013)

31. T. Jacobson, Phys. Rev. Lett. 75, 1260 (1995)

32. C. Eling, R. Guedens, T. Jacobson, Phys. Rev. Lett. 96, 121301 (2006)

33. B. Wang, E. Abdalla, R.K. Su, Phys. Lett. B 503, 394 (2001)

34. B. Wang, E. Abdalla, R.K. Su, Mod. Phys. Lett. A 17, 23 (2002)

35. R.G. Cai, Y.S. Myung, Phys. Rev. D 67, 124021 (2003)

36. A.V. Frolov, L. Kofman, JCAP 0305, 009 (2003). arXiv:hep-th/0212327

37. T. Padmanabhan, Phys. Rep. 406, 49 (2005)

38. A. Paranjape, S. Sarkar, T. Padmanabhan, Phys. Rev. D 74, 104015 (2006). arXiv:hep-th/0607240

39. R.G. Cai, S.P. Kim, JHEP 0502, 050 (2005)

40. M. Akbar, R.G. Cai, Phys. Rev. D 75, 084003 (2007)

41. R.G. Cai, L.M. Cao, Phys. Rev. D 75, 064008 (2007)

42. R.G. Cai, L.M. Cao, Nucl. Phys. B 785, 135 (2007)

43. M. Akbar, R.G. Cai, Phys. Lett. B 648, 243 (2007)

44. A. Sheykhi, B. Wang, R.G. Cai, Nucl. Phys. B 779, 1 (2007)

45. A. Sheykhi, B. Wang, R.G. Cai, Phys. Rev. D 76, 023515 (2007)

46. T. Padmanabhan, Rep. Prog. Phys. 73, 046901 (2010)

47. A. Sheykhi, B. Wang, Phys. Lett. B 678, 434 (2009)

48. A. Sheykhi, Class. Quantum Gravity 27, 025007 (2010)

49. M. Jamil, E.N. Saridakis, M.R. Setare, Phys. Rev. D 81, 023007 (2010). arXiv:0910.0822

50. M. Jamil, E.N. Saridakis, M.R. Setare, JCAP 1011, 032 (2010). arXiv: 1003.0876

51. E.M. Barboza Jr., R.D.C. Nunes, E.M.C. Abreu, J. Ananias Neto, Physica A 436, 301 (2015)

52. R.C. Nunes, E.M. Barboza, E.M.C. Abreu, J.A. Neto, JCAP 1608(08), 051 (2016)

53. G. Kaniadakis, Physica A 296, 405 (2001)

54. G. Kaniadakis, Phys. Rev. E 66, 056125 (2002)

55. E.M.C. Abreu, J.A. Neto, E.M. Barboza Jr., R.C. Nunes, Europhys. Lett. 114, 55001 (2016)

56. A. Lymperis, E.N. Saridakis, Eur. Phys. J. C 78, 993 (2018). arXiv: 1806.04614

57. A. Sheykhi, Phys. Lett. B 785, 118 (2018). arXiv:1806.03996

58. S. Nojiri, S.D. Odintsov, E.N. Saridakis, Eur. J. Phys. C 79, 242 (2019). arXiv: 1903.03098

59. S. Nojiri, S.D. Odintsov, E.N. Saridakis, R. Myrzakulov, Nucl. Phys. B 950, 114850 (2020). arXiv: 1911.03606

60. S.A. Hayward, S. Mukohyana, M.C. Ashworth, Phys. Lett. A 256, 347 (1999)

61. S.A. Hayward, Class. Quantum Gravity 15, 3147 (1998)

62. E. Verlinde, JHEP 1104, 029 (2011)

63. T. Padmanabhan, Mod. Phys. Lett. A 25, 1129 (2010)

64. N.P. Vogt et al., Astron. J. 127, 3273 (2004)

65. P.D. Mannheim, J. O’Brien, Phys. Rev. D 85, 124020 (2010). arXiv: 1011.3495

66. J.G. O'Brien, P.D. Mannheim, Mon. Not. R. Astron. Soc. 421, 1273 (2012). arXiv:1107.5229 